\title{
ON GROUPS WITH A CENTRAL AUTOMORPHISM OF INFINITE ORDER
}

\author{
MARTYN R. DIXON AND M. J. EVANS
}

(Communicated by Warren J. Wong)

\begin{abstract}
It is shown that a group $G$, whose center has finite exponent, has a central automorphism of infinite order if and only if $G$ has an infinite abelian direct factor. It is also shown that the group of central automorphisms of a nilpotent $p$-group of infinite exponent contains an uncountable torsionfree abelian subgroup
\end{abstract}

\section{INTRODUCTION}

In their paper [5] Menegazzo and Stonehewer show that, apart from a few obvious exceptions, a nilpotent $p$-group always has an outer automorphism of order $p$. They also observe that it is often easier, in the case of nilpotent $p$-groups, to construct automorphisms of finite order and, therefore, pose the question of which nilpotent $p$-groups have such an automorphism. A partial answer to this question is stated in [5] and an example of a nilpotent $p$-group that has no automorphism of infinite order is given.

The purpose of the current paper is to characterize those nilpotent $p$-groups $G$ that have a central automorphism of infinite order. (Here an automorphism of $G$ is called central if it acts trivially on the group modulo its center. We denote the group of central automorphisms of $G$ by $A_{\text {ut }} G$.) There are two cases to consider. We show in $\S 2$ that if $G$ is a nilpotent $p$-group of infinite exponent, then $\operatorname{Aut}_{\mathrm{c}} G$ contains an uncountable torsionfree abelian subgroup. The arguments used here follow those of Buckley and Wiegold [1, Theorems 2.2, 2.6], for the most part. However, some additional results are needed, because the automorphisms constructed in [1] do not always have infinite order. In $\S 3$ we obtain our main result concerning central automorphisms of nilpotent $p$ groups. We show that if $G$ is such a group of finite exponent, then $G$ has a central automorphism of infinite order if and only if $G$ has an infinite abelian direct factor. However, our result is a corollary to the following much more general theorem, which we prove in $\S 3$.

Theorem 3.1. Suppose $G$ is a group and $Z(G)$ has finite exponent. Then the following are equivalent.

(i) $G$ has a central automorphism of infinite order.

Received by the editors August 31, 1990.

1980 Mathematics Subject Classification (1985 Revision). Primary 20E36, 20 F28. 
(ii) $G$ has an infinite abelian direct factor.

(iii) $\mathrm{Aut}_{\mathrm{c}} G$ contains an uncountable torsionfree abelian subgroup.

Our notation is standard for the most part. For any group $G$, the commutator subgroup is $G^{\prime}$, the center is $Z(G)$, which we abbreviate to $Z$, and we write $G_{a b}$ for $G / G^{\prime}$. We use additive notation for abelian groups and, in particular, for subgroups of $Z$. For a prime $p$ and natural number $n, Z\left[p^{n}\right]$ denotes the subgroup of elements of $Z$ of order dividing $p^{n}$, and $p^{n} Z$ denotes the subgroup of $Z$ consisting of $\left\{p^{n} z \mid z \in Z\right\}$. We also recall that if $N \leq Z$ then $\operatorname{Hom}(G / N, N)$ can be identified with the subgroup of elements of $\operatorname{Aut}_{\mathrm{c}} G$ that act trivially on both $N$ and $G / N$.

\section{NILPOTENT $p$-GROUPS OF INFINITE EXPONENT}

Throughout this section we let $G$ be a nilpotent $p$-group of infinite exponent. We refer the reader to [1] and [8] for standard facts concerning basic subgroups of nilpotent $p$-groups; recall that a subgroup $B$ of the group $G$ is called basic if $G^{\prime} \leq B$ and $B / G^{\prime}$ is a basic subgroup of the abelian group $G_{\mathrm{ab}}$. We require two preliminary results before proving the main result of this section.

Lemma 2.1. Suppose $B$ is a basic subgroup of $G$ and $N \leq Z(G)$. Suppose $G=B N$ and $B \cap N$ has finite exponent. Then $\operatorname{Aut}_{c} G$ contains an uncountable torsionfree abelian subgroup.

Proof. Let $Q=N / B \cap N$, so $Q$ is a periodic radicable abelian group. Because $B \cap N$ has finite exponent, it follows that $\operatorname{Ext}(Q, B \cap N)$ has finite exponent $m$, say and clearly $m$ is a power of $p$. If $\pi$ is a $p$-adic integer of the form $1+p m+s_{2} p^{2}+s_{3} p^{3}+\ldots$, where each $s_{i}$ is either 0 or $m$ and infinitely many of them are nonzero, then multiplication by $\pi$ induces an automorphism of $Q$ and by [4, Lemma 52.1], $\pi^{*}$ is multiplication by $\pi$ on $\operatorname{Ext}(Q, B \cap N)$. Thus if $\Delta \in \operatorname{Ext}(Q, B \cap N)$,

$$
\pi^{*} \Delta=\Delta \text { because } m \Delta=0 .
$$

On the other hand, because $Q$ is divisible and $B \cap N$ has finite exponent, the theory of automorphisms of group extensions (see [7, p. 70]) shows that

$$
C_{\text {Aut } Q}(\Delta) \leq \text { Aut } N \text {. }
$$

Hence Aut $N$ contains an uncountable, torsionfree abelian subgroup $A$, fixing $B \cap N$. Finally if we define, for each $\alpha \in A$,

$$
(b n) \bar{\alpha}=b(n \alpha) \quad \text { for } b \in B, n \in N \text {, }
$$

then $\bar{\alpha} \in$ Aut $G$ and it follows that Aut $G$ contains a subgroup isomorphic to $A$.

The Cartesian product of a family $\left\{G_{i}: i \in I\right\}$ is denoted by $\mathrm{Cr}_{i \in I} G_{i}$ and the direct sum by $\bigoplus_{i \in I} G_{i}$. For a natural number $n$, we let $C_{n}$ be the cyclic group of order $n$, and for the prime $p$, we let $C_{p \infty}$ be the quasicyclic $p$-group.

Lemma 2.2. Suppose $I$ is an index set and $B=\bigoplus_{i \in I} C_{p^{n(i)}}$. Suppose that $C$ is a subgroup of $B$ of infinite exponent. Then there is a direct sum decomposition $B=X \oplus Y$ such that both $X \cap C$ and $Y \cap C$ have infinite exponent.

Proof. We may assume $D=\bigoplus_{i=1}^{\infty} C_{p^{n(i)}}$, where $n(i) \leq n(j)$ if $i \leq j$, is a subgroup of $B$ such that $E=C \cap D$ has infinite exponent. Let $\left(c_{11}, \ldots, c_{1, k_{1}}\right.$, 
$0, \ldots),\left(c_{21}, \ldots, c_{2}, k_{2}, 0, \ldots\right), \ldots$ be a sequence of elements of $E$ with orders $p^{m_{1}}, p^{m_{2}}, \ldots$ where

$$
p^{m_{i+1}}>p^{n\left(k_{i}\right)+m_{i}} \quad i=1,2, \ldots .
$$

Then $k_{i}<k_{i+1}$ for all $i$. Consider

$$
p^{n\left(k_{i}\right)}\left(c_{i+1,1}, \ldots, c_{i+1, k_{i}}, \ldots, c_{i+1, k_{i+1}}, 0, \ldots\right) .
$$

This is an element of $E$ of order $p^{m_{i+1}-n\left(k_{i}\right)}>p^{m_{i}}$. Set $I_{1}=\left\{1, \ldots, k_{1}\right\}$ and $I_{j+1}=\left\{k_{j}+1, \ldots, k_{j+1}\right\}$ for $j \geq 1$. We may find sets $J, K \subseteq I$ such that $J \cup K=I, J \cap K=\varnothing, \bigcup_{j=1}^{\infty} I_{2 j} \subseteq J$, and $\bigcup_{j=1}^{\infty} I_{2 j-1} \subseteq K$. Then set $X=\bigoplus_{i \in J} C_{p^{n(i)}}$ and $Y=\bigoplus_{i \in K} C_{p^{n(i)}}$. The subgroups $X$ and $Y$ have the desired properties.

Theorem 2.3. If $G$ is a nilpotent p-group of infinite exponent then Aut $_{\mathrm{c}} G$ contains an uncountable torsionfree abelian subgroup.

Proof. There are a number of cases to consider.

Case 1. Suppose $G$ is nonreduced, and let $B$ be a basic subgroup of $G$. Let $N$ be a central subgroup of $G$ such that $N \cong C_{p}$. If $G / B N$ is nontrivial, then $\operatorname{Hom}(G / B N, N)$ is an uncountable torsionfree abelian subgroup of $\operatorname{Aut}_{\mathrm{c}} G$ as required. If $G=B N$ then either $N \leq B$ or $B \cap N$ is finite. In the former case, $G_{\mathrm{ab}}=\bigoplus_{i \in I} C_{p^{n(i)}}$, for some index set $I$ and $G_{\mathrm{ab}}$ has infinite exponent, since $G$ does. Since $G_{\mathrm{ab}}$ is reduced, $N \leq G^{\prime}$ and hence $\operatorname{Hom}\left(G_{\mathrm{ab}}, N\right) \leq \operatorname{Aut}_{\mathrm{c}} G$. Thus $\mathrm{Cr}_{i \in I} N\left[p^{n(i)}\right]$ is a subgroup of $\operatorname{Aut}_{\mathrm{c}} G$ and the result now follows from [1, Lemma 2.5]. If $B \cap N$ is finite then Lemma 2.1 applies, again giving the result.

Case 2. Suppose $G$ is reduced. According to [8, XVI] every basic subgroup $B$ is infinite. If $B$ has finite exponent then $[8, \mathrm{XV}]$ shows that $G=B Z$. Hence by Lemma 2.1, Aut $G$ satisfies the desired conclusion. If $B$ has infinite exponent but $G^{\prime} \cap Z$ has finite exponent, then $G / Z$ also has finite exponent (the correct version of $[8, \mathrm{IX}])$. Hence $B \cap Z$ has infinite exponent. Then $(B \cap Z) G^{\prime} / G^{\prime}$ is a subgroup of infinite exponent in the group $B / G^{\prime}$, a direct sum of cyclic groups. By Lemma 2.2, we can find $X / G^{\prime}, Y / G^{\prime}$ so that $B / G^{\prime}=X / G^{\prime} \oplus Y / G^{\prime}$ and $X / G^{\prime} \cap(B \cap Z) G^{\prime} / G^{\prime}, Y / G^{\prime} \cap(B \cap Z) G^{\prime} / G^{\prime}$ both have infinite exponent.

Hence $X \cap Z$ and $Y \cap Z$ both have infinite exponent. However, $B / X$ is a basic subgroup of $G / X$ and the epimorphism of Szele [4, 36.1] yields

$$
\operatorname{Hom}\left(Y / G^{\prime}, X \cap Z\right) \leq \operatorname{Hom}(G / X \cap Z, X \cap Z) \leq \operatorname{Aut}_{\mathrm{c}} G
$$

and Aut $_{\mathrm{c}} G$ contains an uncountable torsionfree abelian subgroup in this case.

Finally, if both $B$ and $G^{\prime} \cap Z$ have infinite exponent then the proof of [1, Theorem 2.6] gives the result in this case. This completes the proof.

\section{THE FINITE EXPONENT CASE}

We first give a proof of Theorem 3.1.

Let $\pi$ denote the set of primes dividing the exponent of $Z(G)$.

(i) implies (ii). Let $\alpha$ be a central automorphism of $G$ that has infinite order and note that $\alpha$ induces an automorphism of infinite order on $Z=Z(G)$. (We say that $\alpha$ acts infinitely on $Z$.) Since $\pi$ is finite and, for each prime $p$, the Sylow $p$-subgroups of $Z$ are characteristic in $G$, there is some prime $p \in \pi$ 
such that $\alpha$ acts infinitely on the Sylow $p$-subgroup of $Z$, which we denote by $K$. Let $L$ be the $p^{\prime}$-part of $Z$ so that $Z=K \oplus L$.

Let $k \in \mathbb{N}$ be minimal such that some nontrivial power of $\alpha$ acts trivially on $p^{k} K$. Replacing $\alpha$ with a nontrivial power of itself if necessary we may assume that $\alpha$ acts trivially on $p^{k} K$. It then follows that $\alpha$ acts infinitely on $p^{k-1} K / p^{k} K$, otherwise some nontrivial power of $\alpha$ acts trivially on both $p^{k-1} K / p^{k} K$ and $p^{k} K$. However, this implies some nontrivial power of $\alpha$ acts trivially on $p^{k-1} K$, contrary to the minimality of $k$.

We set $M=p^{k-1} K(\alpha-1)=\left\{z^{\alpha}-z \mid z \in p^{k-1} K\right\}$ and note that $M$ is an $\alpha$-invariant subgroup of $p^{k-1} K$. To complete the proof we now establish a series of claims.

(a) $M$ has exponent $p$.

For if $m \in M$, then $m=z^{\alpha}-z$ for some $z \in p^{k-1} K$. Then $p z \in p^{k} K$ so

$$
p z=(p z) \alpha=p(z \alpha)=p z+p m \text {. }
$$

Hence $p m=0$ and (a) follows.

(b) $\alpha$ acts infinitely on $M$.

For if $\alpha$ acts finitely on $M$ then some nontrivial power of $\alpha$ acts trivially on $M$. Clearly $\alpha$ acts trivially on $p^{k-1} K / M$ so some power of $\alpha$ acts trivially on $p^{k-1} K$, which contradicts the choice of $k$.

(c) $\alpha$ acts infinitely on $P \equiv M G^{\prime}\left(p^{k} K\right) / G^{\prime}\left(p^{k} K\right)$.

Otherwise $\alpha$ acts finitely on $P$, so some nontrivial power of $\alpha$ acts trivially on $P$. Since $\alpha$ is central, it acts trivially on $G^{\prime}$. Hence some nontrivial power of $\alpha$ acts trivially on $M G^{\prime}\left(p^{k} K\right)$, contrary to (b). Hence (c) follows.

We let $C=C_{p}(\alpha)$. It follows from (a) that $P=C \oplus D$ for some subgroup $D$ (which is not necessarily $\alpha$-invariant.) Furthermore $D$ is infinite by (c). Let $I$ be an index set and, for $i \in I$, choose $r_{i} \in M$ so that $\left\{r_{i} G^{\prime}\left(p^{k} K\right): i \in I\right\}$ is a basis of $D$. Define $N=\left\langle r_{i}: i \in I\right\rangle$ and note that $N$ is an infinite elementary abelian subgroup of $M \leq p^{k-1} K$. It is clear that:

(d) $n^{\alpha} \not \equiv n \bmod G^{\prime}\left(p^{k} K\right)$ for all nontrivial $n \in N$.

It follows from [4, p. 119, Example 5] that $K$ (and hence $Z$ ) has a direct summand $A$ such that $A[p]=N$. Set $Z=A \oplus F$. Since $A$ is a direct summand of $Z$, each $0 \neq n \in N$ has the same $p$-height in $A$ as in $Z$. Furthermore $N \leq M \leq p^{k-1} K$. Hence:

(e) The p-height of $0 \neq n \in N$ in $A$ is at least $k-1$. (In fact one can easily see that it is exactly $k-1$.)

(f) $A G^{\prime} / G^{\prime}$ is a direct summand of $G_{a b}$.

It suffices by [4, Corollary 27.5] to show that $A G^{\prime} / G^{\prime}$ is pure in $G_{\mathrm{ab}}$, and to do this it is enough to show that every element of order $p$ in $A G^{\prime} / G^{\prime}$ has the same $p$-height in $G_{\text {ab }}$ as in $A G^{\prime} / G^{\prime}$ (see [4, p. 114, (h)] and also note that the Sylow $p$-subgroup of $G_{\mathrm{ab}}$ is pure in $G_{\mathrm{ab}}$ ).

Now $A \cap G^{\prime}=1$ since $\alpha$ fixes no nontrivial element of $A[p]=N$, by (d), whereas the central automorphism $\alpha$ acts trivially on $G^{\prime}$. It follows that $\left(A G^{\prime} / G^{\prime}\right)[p]=N G^{\prime} / G^{\prime}$. Suppose for a contradiction that $n+G^{\prime} \in N G^{\prime} / G^{\prime}$ has larger $p$-height in $G_{\mathrm{ab}}$ than in $A G^{\prime} / G^{\prime}$. Then by (e), $n+G^{\prime}=p^{l} g+G^{\prime}$ for some $g \in G$ and some $l \geq k$. Since $\alpha$ is central, $g \alpha=g+z$ for some $z \in Z$ so that

$$
n \alpha+G^{\prime}=\left(p^{l} g\right) \alpha+G^{\prime}=p^{l} g+p^{l} z+G^{\prime}=n+p^{l} z+G^{\prime}
$$


Also $p n=0$ so $p^{l+1} g \in G^{\prime}$ and since $\alpha$ is central $p^{l+1} g=\left(p^{l+1} g\right) \alpha=$ $p^{l+1} g+p^{l+1} z$. Hence $z \in K$, the Sylow $p$-subgroup of $Z$ so (1) shows

$$
n \alpha \equiv n \bmod G^{\prime} p^{k} K,
$$

contrary to $(\mathrm{d})$. This proves (f).

The result now follows immediately. For there is a subgroup $H$ of $G$ such that

$$
G_{\mathrm{ab}}=A G^{\prime} / G^{\prime} \oplus H / G^{\prime} .
$$

Hence $G=A H$. But $A \cap H \leq A \cap G^{\prime}=1$ and $A \leq Z(G)$ so it follows that $G=A \times H$.

(ii) implies (iii). Clearly $\operatorname{Cr}_{m=1}^{\infty} C_{p^{m}} \leq \operatorname{Aut}_{\mathrm{c}} G$. Hence by [1, Lemma 2.5] the result follows.

(iii) implies (i). This is clear.

In particular, we have

Corollary 3.2. Suppose $G$ is a nilpotent p-group of finite exponent. Then $G$ has a central automorphism of infinite order if and only if $G$ has an infinite abelian direct factor.

This result is analogous to [3, Lemma 2] although the proof is rather different. The reader is also referred to $[2,6]$ where further results have been obtained on central automorphisms of infinite groups.

An example. We note that Theorem 3.1 fails if $Z(G)$ is allowed to have infinite exponent. For each prime $p$, let $G_{p}$ be a nilpotent $p$-group as in [5, 3.2(ii)] such that Aut $G_{p}=\operatorname{Aut}_{c} G_{p}$ is an elementary abelian $p$-group. Set $G=\bigoplus G_{p}$, the direct sum being taken over all primes $p$. Then Aut $G=\operatorname{Cr}\left(\operatorname{Aut} G_{p}\right)$ and Aut $_{\mathrm{c}} G$ clearly contains elements of infinite order. However $G$ has no infinite abelian direct factor.

\section{ACKNOWLEDGMENT}

The first author is indebted to the Mathematics Institute, University of Warwick for its hospitality while this research was being done.

\section{REFERENCES}

1. J. Buckley and J. Wiegold, On the number of outer automorphisms of an infinite nilpotent p-group, Arch. Math. 31 (1978), 321-328.

2. M. Curzio, D. J. S. Robinson, H. Smith, and J. Wiegold, Some remarks on central automorphisms of hypercentral groups, Arch. Math. 53 (1989), 327-331.

3. S. Franciosi and F. de Giovanni, On torsion groups with nilpotent automorphism groups, Comm. Algebra 14 (1986), 1909-1935.

4. L. Fuchs, Infinite abelian groups, vol. 1, Academic Press, New York and London, 1970.

5. F. Menegazzo and S. E. Stonehewer, On the automorphism group of a nilpotent p-group, J. London Math. Soc. (2) 31 (1985), 272-276.

6. M. R. Pettet, Central automorphisms of periodic groups, Arch. Math. 51 (1988), 20-33.

7. D. J. S. Robinson, Applications of cohomology to the theory of groups, Groups-St. Andrews 1981, London Mathematical Society Lecture Note series, vol. 71, Cambridge Univ. Press, Cambridge, 1982. 
8. A. E. Zaleskiī, A nilpotent p-group has an outer automorphism, Dokl. Akad. Nauk SSSR 196 (1971); Soviet Math. Dokl. 12 (1971), 227-230.

Department of Mathematics, University of Alabama, Tuscaloosa, Alabama 35487 0350 